\title{
A Modified Method for Examining the Walking Pattern and Pace of COPD Patients in a 6-min Walk Test Before and After the Inhalation of Procaterol
}

\author{
Naoto Burioka ${ }^{1}$, Sachiko Nakamoto ${ }^{1}$, Takashi Amisaki ${ }^{2}$, Takuya Horie ${ }^{1}$ and Eiji Shimizu ${ }^{3}$
}

\begin{abstract}
Objective The 6-min walk test (6MWT) is a simple test that is used to examine the exercise tolerance and outcomes in patients with chronic obstructive pulmonary disease (COPD). Although the 6MWT is useful for assessing exercise tolerance, it is difficult to evaluate time-dependent parameters such as the walking pattern. A modified 6MWT has been devised to assess the walking pattern by calculating the number of steps per second (NSPS). This study was performed to investigate walking pattern of COPD patients in the modified $6 \mathrm{MWT}$ before and after a single inhalation of the short-acting $\beta_{2}$-agonist procaterol.

Methods Nine male COPD patients participated in this study. The 6MWT was performed before and after the inhalation of procaterol hydrochloride. A digital video recording of the 6MWT was made. After the 6MWT, the number of steps walked by the subject in each 5-s period was counted manually with a hand counter while viewing the walking test on the video monitor.

Results After the inhalation of procaterol, the 6-min walking distance increased significantly in comparison to baseline $(\mathrm{p}<0.01)$. The mean NSPS was also significantly increased after the inhalation of procaterol in comparison to baseline $(\mathrm{p}<0.01)$. The walking pattern was displayed on a graph of time versus NSPS, and the walking pace was shown by a graph of time versus cumulative steps.

Conclusion The analysis of the COPD patients' walking test performance and their walking pattern and pace in the 6MWT may help to evaluate the effects of drug treatment.
\end{abstract}

Key words: $\beta_{2}$-agonist, chronic obstructive pulmonary disease, 6-min walk test, procaterol, steps

(Intern Med 56: 1949-1955, 2017)

(DOI: 10.2169/internalmedicine.56.7961)

\section{Introduction}

The 6-min walk test (6MWT) has been used to evaluate the functional capacity and to predict mortality in patients with chronic respiratory disease $(1,2)$. This test assesses the maximum distance that a patient can walk during a 6-min period (3). Although the test is simple, it is useful for measuring clinical improvement in response to pulmonary rehabilitation and drug therapy (4).

Chronic obstructive pulmonary disease (COPD) is one of the most important respiratory diseases and is associated with a high mortality rate (5). Physical activity and the number of steps walked per day are strong predictors of allcause mortality in COPD patients $(6,7)$. The functional status of COPD patients is examined by performing lung function tests and timed walking tests such as the 6MWT. In COPD patients, exercise causes dynamic lung hyperinflation and an increase in the end-expiratory lung volume (EELV) due to the trapping of air due to decreased elastic recoil, which occurs secondarily to the destruction of the alveoli and the narrowing of the small airways (8-10). The inspiratory capacity (IC) of COPD patients is significantly decreased during the 6MWT (11). The decrease in the IC of

\footnotetext{
${ }^{1}$ Department of Pathobiological Science and Technology, School of Health Science, Tottori University Faculty of Medicine, Japan, ${ }^{2}$ Department of Biological Regulation, School of Health Science, Tottori University Faculty of Medicine, Japan and ${ }^{3}$ Division of Medical Oncology and Molecular Respirology, Department of Multidisciplinary Internal Medicine, School of Medicine, Tottori University Faculty of Medicine, Japan Received for publication July 7, 2016; Accepted for publication October 4, 2016 Correspondence to Dr. Naoto Burioka, burioka@med.tottori-u.ac.jp
} 
Table 1. Pulmonary Function of Male COPD Patients $(\mathbf{n}=9)$.

\begin{tabular}{lcc}
\hline & before & after procaterol inhalation \\
\hline FVC (L) & $2.56 \pm 0.73$ & $2.80 \pm 0.77$ \\
$\mathrm{FEV}_{1}(\mathrm{~L})$ & $1.21 \pm 0.52$ & $1.38 \pm 0.68$ \\
$\mathrm{FEV}_{1} / \mathrm{FVC}(\%)$ & $46.7 \pm 12.3$ & $47.5 \pm 13.3$ \\
FEV $_{1} \%$ pred. (\%) & $56.6 \pm 25.2$ & $62.0 \pm 28.1$ \\
\hline
\end{tabular}

Values are the mean \pm standard deviation.

FVC: forced vital capacity

$\mathrm{FEV}_{1}$ : forced expiratory volume in one second

COPD patients was significantly attenuated and the 6-min walking distance (6MWD) was increased after the inhalation of the short-acting $\beta_{2}$-agonist procaterol hydrochloride in comparison to patients who inhaled a placebo (11).

However, many COPD patients cannot walk for 6 minutes without stopping and may have to rest several times during the 6MWT due to dyspnea (12). Their walking pace and resting time may influence the results of the 6MWT, because oxygen desaturation can be improved by resting. Although the conventional 6MWT is useful for assessing the general exercise tolerance, it is difficult to evaluate timedependent parameters such as the instantaneous walking speed. A modified 6MWT has been developed to examine the walking pattern by determining the number of steps per second (NSPS) (12). In the present study, we assessed the effect of a single dose of inhaled procaterol on the improvement of the walking distance, the resting time, and the walking pattern and pace (as NSPS) in COPD patients who performed the 6MWT.

\section{Materials and Methods}

\section{Patients}

Nine male patients with COPD (72.9 \pm 6.0 years) participated in this study. Spirometry was performed before and after the inhalation of $20 \mu \mathrm{g}$ of procaterol hydrochloride (Meptin $^{\mathrm{TM}}$, Otsuka Pharmaceutical, Tokyo, Japan) using a pulmonary function testing system (Chestac-7800, Chest, Tokyo, Japan) on another day (Table 1). The severity of airflow limitation in COPD was classified according to forced expiratory volume in one second $\left(\mathrm{FEV}_{1}\right)$ after the inhalation of a bronchodilator (5). Five of the 9 patients inhaled tiotropium, 3 patients inhaled tiotropium and salmeterol, and 1 patient inhaled formoterol with budesonide, regularly. Patients who had experienced unstable angina or myocardial infarction during the previous month, those who had a resting heart rate of more than $120 \mathrm{bpm}$, a resting systolic blood pressure of more than $180 \mathrm{mmHg}$ or a diastolic pressure of more than $100 \mathrm{mmHg}$, orthopedic or neurologic conditions were excluded from the study (3). The present study was approved by the ethics boards of Tottori University and Hitachi Memorial Hospital (No. 1845). All of the participants gave their written informed consent.

\section{Study protocol}

The baseline 6MWT was performed before the inhalation of procaterol. The subjects performed the baseline 6MWT and then inhaled $20 \mu \mathrm{g}$ of procaterol using a spacer device. After resting on a chair for 20 minutes, the patients performed the 6MWT again. The 6MWT was performed in a flat corridor of $54 \mathrm{~m}$ in length at Tottori University Hospital or Hitachi Memorial Hospital; the other technical aspects of the 6MWT were in accordance with the published guidelines (3). Arterial blood oxygen saturation was measured by pulse oximetry $\left(\mathrm{SpO}_{2}\right)$ with a finger sensor (Pulsox 300i, Konica-Minolta, Tokyo, Japan) and was continuously recorded during the test. The pulse oximetry variables were analyzed using the DS-5 Pulsox software program (KonicaMinolta). The severity of dyspnea was subjectively assessed before and after the 6MWT using a modified Borg scale (13). It has been reported that at least 2 practice walks should be performed before the 6MWT since training influences the results of the test $(14,15)$. The patients performed at least 2 practice walks on another day prior to the actual test because dyspnea and fatigue could have influenced the results of the 6MWT if they had practiced on the same day. The patients were allowed to sit on a chair to rest during the test, if needed, and resumed walking themselves when they had recovered. The duration of each rest was measured with a stopwatch. All of the walking tests were recorded on digital video and the 6MWD was measured.

\section{The calculation of the number of steps per second (NSPS)}

The NSPS is a new index (12); it is defined as the steps walked in $A$-second period divided by $A$, where $A$ is a divisor of 360 . We used $A=5$ (5-s interval) in the present study. The mean NSPS is calculated using the following formula:

$$
\begin{aligned}
& \text { Mean NSPS }=\frac{\lfloor\text { total steps in } 360\rfloor}{360}=\frac{\lfloor\text { total steps in } 360\rfloor \div 5}{360 \div 5} \\
& =\frac{\{\text { steps in } 0<t \leq 5\rfloor+\lfloor\text { steps in } 5<t \leq 10\rfloor+\cdots+\lfloor\text { steps } \text { in } 355<t \leq 360\rfloor\} \div 5}{72} \\
& =\frac{\sum_{k=1}^{72}\{\lfloor\text { steps in } 5(k-1)<t \leq 5 k\rfloor \div 5\}}{72}=\frac{\sum_{k=1}^{72} N S P S_{k}}{72},
\end{aligned}
$$

where $L\rfloor$ is the floor function, and $\operatorname{NSPS}_{k}$ is the value of NSPS at the $k$-th 5 -s interval $(\mathrm{k}=1,2,3, \ldots, 72) . N S P S_{k}$ shows the slope of time versus the cumulative steps in 5-s (12).

The number of steps walked by a subject in each 5-s period was counted manually with a hand counter while viewing the walking test on the video monitor. The walking speed $(\mathrm{m} / \mathrm{s})$ was considered to be the average step length $(\mathrm{m} / \mathrm{step}) \times$ NSPS $(\mathrm{step} / \mathrm{s})(12)$. The NSPS was calculated as the number of steps walked in a 5 -s period divided by 5 , and this calculation was performed for 72 consecutive periods $(360 \mathrm{~s} \div 5 \mathrm{~s})$ in each subject. Because the NSPS is proportional to the walking speed, it usually decreases when a patient walks more slowly and falls to zero if the patient 
Table 2. Results of the 6-min Walk Test (6MWT) before and after Inhalation of Procaterol (n=9).

\begin{tabular}{lccc}
\hline & First 6MWT & $\begin{array}{l}\text { Second 6MWT after } \\
\text { procaterol inhalation }\end{array}$ & p value \\
\hline 6-min distance $(\mathrm{m})$ & $258.7 \pm 124.0$ & $293.4 \pm 115.6$ & $\mathrm{p}<0.01 *$ \\
Total no. of steps & $473.6 \pm 153.9$ & $526.1 \pm 135.6$ & $\mathrm{p}<0.02 *$ \\
Resting HR before test $\left(\mathrm{min}^{-1}\right)$ & $91.5 \pm 10.5$ & $92.3 \pm 9.1$ & $\mathrm{p}>0.43 *$ \\
Mean length of a step $(\mathrm{m})$ & $0.52 \pm 0.09$ & $0.54 \pm 0.09$ & $\mathrm{p}>0.10^{*}$ \\
Mean NSPS (step/s) & $1.32 \pm 0.43$ & $1.46 \pm 0.38$ & $\mathrm{p}<0.01 *$ \\
Total resting time (s) & $85(20.3-149.8) \dagger$ & $59(13.3-104.8) \dagger$ & $\mathrm{p}<0.05 * *$ \\
Borg scale after 6MWT & $3.0(1.8-4.2) \dagger$ & $3.0(1.9-4.1) \dagger$ & $\mathrm{p}>0.99 * *$ \\
Lowest $\mathrm{SpO}_{2}(\%)$ & $88.7 \pm 5.1$ & $88.2 \pm 5.5$ & $\mathrm{p}>0.45^{*}$ \\
${\text { Mean } \mathrm{SpO}_{2}(\%)}^{91.4 \pm 3.7}$ & $91.3 \pm 3.8$ & $\mathrm{p}>0.75 *$ \\
\hline
\end{tabular}

\section{HR: heart rate}

Values are the mean \pm standard deviation or median (interquartile range) $\dagger$.

*: Paired t-test, **: Wilcoxon matched-pairs signed-rank test
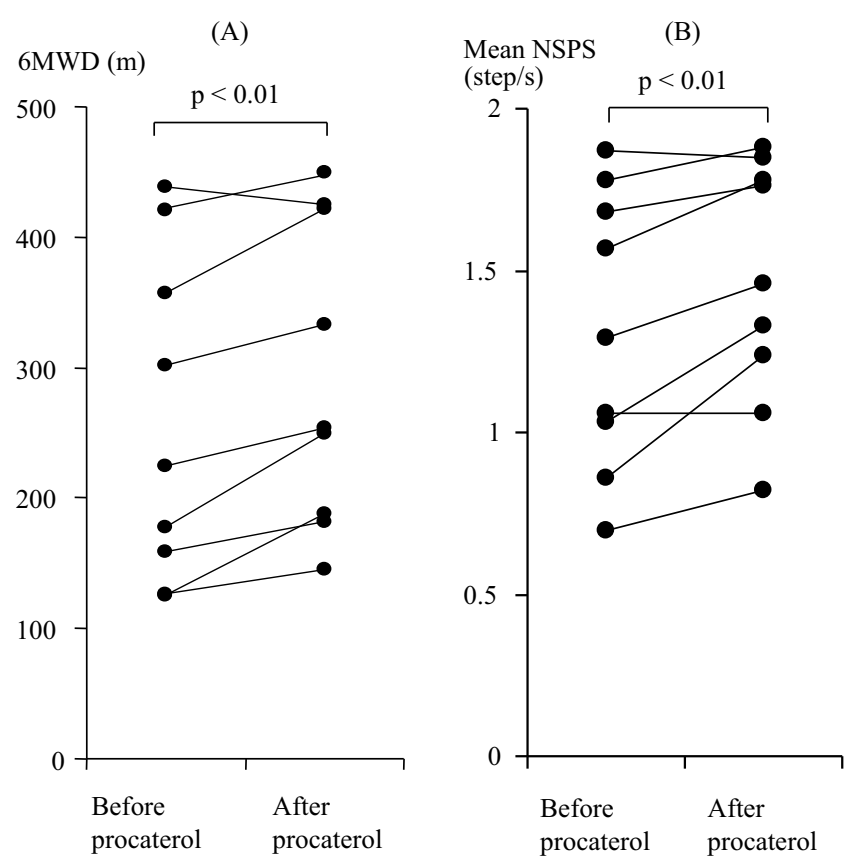

Figure 1. The 6-min walking distance (6MWD) and the mean of number of steps per second (NSPS) before and after the inhalation of procaterol. (A) The inhalation of procaterol significantly increased the mean 6MWD. (B) The mean NSPS was also significantly increased after the inhalation of procaterol.

stops walking (12).

\section{Statistical analysis}

The results are presented as the median (interquartile range) or mean $\pm \mathrm{SD}$. Comparisons were performed using the Wilcoxon matched-pairs signed-rank test or the paired $t$ test. The StatFlex software program was used to perform the statistical analyses (StatFlex, ViewFlex, Tokyo, Japan). p values of $<0.05$ were considered to indicate statistical significance.

Results

With regard to the severity of airflow limitation in our COPD patients, 3 patients were classified as Global initiative for chronic Obstructive Lung Disease (GOLD) 1 (predicted $\left.\mathrm{FEV}_{1}(\%) \geq 80 \%\right), 2$ patients were classified as GOLD $2(50 \%$ to $<80 \%), 3$ patient were GOLD $3(30 \%$ to $<50 \%)$, and 1 patient was classified as GOLD $4(<30 \%)(5)$. The height, weight and body mass index of the 9 COPD patients were $1.58 \pm 0.06 \mathrm{~m}, 51.6 \pm 9.2 \mathrm{~kg}$ and $20.7 \pm 4.2 \mathrm{~kg} / \mathrm{m}^{2}$, respectively. Table 1 shows the results of a pulmonary function test before and after the inhalation of procaterol on another day. Table 2 shows the results of the 6MWT before and after the inhalation of procaterol. After a single inhalation of procaterol, the 6MWD was significantly longer in comparison to baseline (Fig. 1A). An increase of $>30 \mathrm{~m}$ in the 6MWD is considered to be the minimal clinical important difference (MCID) for the patients with chronic respiratory disease $(1,2)$. After the inhalation of procaterol, the 6MWD changed by more than $30 \mathrm{~m}$ in 4 of the 9 COPD patients. The resting heart rate was not significantly changed in comparison to that before the 6MWT. The total number of steps in 6 minutes was significantly increased by the inhalation of procaterol, but the mean step length did not change (Table 2). Six of the 9 patients had to rest on a chair during the baseline 6MWT. The median (range) number of rests before and after the inhalation of procaterol was $1(0-3)$ and 1 (0$3)$, respectively. Although the number of rests was almost same, the total resting time was significantly shorter after the inhalation of procaterol (Table 2). The mean NSPS showed a significant increase after the inhalation of procaterol in comparison to the baseline value (Fig. 1B). On the other hand, the lowest $\mathrm{SpO}_{2}$ and mean $\mathrm{SpO}_{2}$ values were not significantly different after the inhalation of procaterol. There was no significant difference in the modified Borg scale values after the 6MWT before and after the inhalation of procaterol (Table 2).

Fig. 2A shows an example of the walking pattern and 

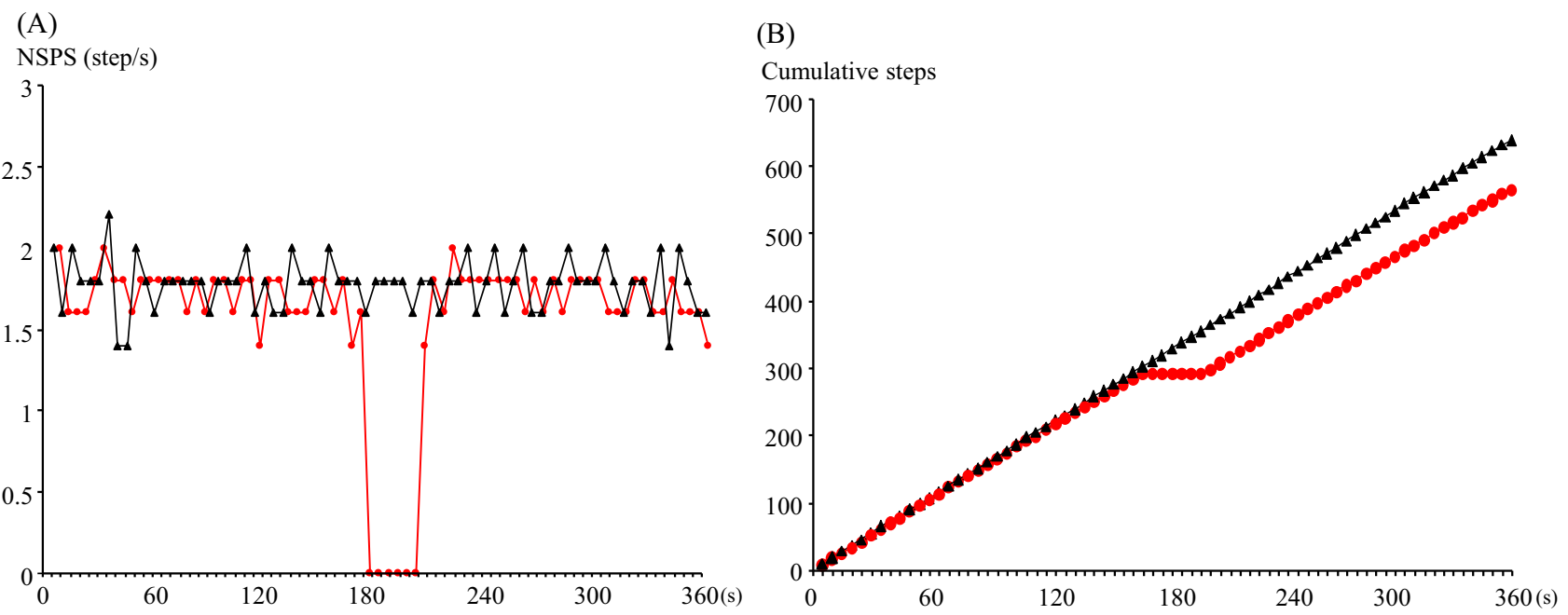

Figure 2. Determining the walking pattern and pace from the number of steps per second (NSPS) and the cumulative steps before and after the inhalation of procaterol. The patient was a 73-year-old man with COPD. Although the patient walked $302 \mathrm{~m}$ in the 6-min walk test (6MWT) with $32 \mathrm{~s}$ of rest before the inhalation of procaterol (closed circle), he could walk $332.5 \mathrm{~m}$ without a rest after the inhalation of procaterol (closed triangle). The walking pattern is clearly displayed on this graph of time versus NSPS (A). During the 6MWT, the NSPS falls to zero each time the patient stops walking. The cumulative number of steps is shown as a function of time for each test $(B)$. The slope indicates the walking pace. This patient walked at the same pace before and after the inhalation of procaterol, except for during rest periods.

pace (as the NSPS) in a 73-year-old patient with moderate COPD. The 6MWD improved by $30.5 \mathrm{~m}$ following the inhalation of procaterol. Note that NSPS falls to zero when the patient stops walking. The patient rested for a total of 32-s before the inhalation of procaterol (Fig. 2A). After the inhalation of procaterol, this patient could walk for 6 minutes without rest. Fig. $2 \mathrm{~B}$ shows the numbers of the cumulative steps during the 6MWT for the same patient. The results of the two tests (before and after the inhalation of procaterol) shared a common slope (except for during the rest periods), and the slope was constant (Fig. 2B), which suggested that, with the exception of the rest periods, the walking pace was almost the same during the 6MWT. The mean NSPS before and after the inhalation of procaterol in the 6MWT was 1.57 and 1.78 (step/s), respectively. The mean NSPS is increased by a decrease in the rest time and/or an increase in the walking pace. In this case, the inhalation of procaterol led to a decrease in the rest time, resulting in an increase in the mean NSPS. Figs. 3 and 4 show that when the resting time was decreased by the inhalation of procaterol, the 6MWD was improved by more than $30 \mathrm{~m}$ after the inhalation of procaterol.

Fig. 5A shows an example of the increase in the walking pace after the inhalation of procaterol. This 68-year-old patient could walk in 6-minute without a rest. Fig. 5B shows a graph of time versus the cumulative steps during the 6MWT. The slope increased after the inhalation of procaterol (Fig. 5B), which suggests that the walking pace increased during the 6MWT. The mean NSPS before and after the inhalation of procaterol in the 6MWT was 1.78 and 1.88 (step/s), respectively. In this case, the increase in the walking pace following the inhalation of procaterol might have resulted in an increase in the mean NSPS; however, a placebo study will be necessary. The results in 5 participants in whom the improvement in the 6MWD was $<30 \mathrm{~m}$ after the inhalation of procaterol are not shown.

\section{Discussion}

The present study demonstrated that a single inhalation of procaterol significantly prolonged the $6 \mathrm{MWD}$, and shortened the total resting time or increased the walking pace during the 6MWT, suggesting that the inhalation of procaterol may be useful for improving the exercise performance of COPD patients undertaking this test. In addition, the steps per second and the cumulative steps were effective measures for analyzing the changes in the walking pattern and pace after the inhalation of procaterol in COPD patients. Determining the number of steps walked per unit time and the consideration of the total resting time during the 6MWT may be important when assessing the functional status before and after drug treatment because some COPD patients cannot walk continuously for 6-minute without resting.

In COPD patients, exercise causes an increase in the EELV along with an increase of ventilation, which is known as dynamic lung hyperinflation (8-10). A significant relationship has been reported between dynamic lung hyperinflation and dyspnea during exercise in COPD patients (8). Several reports have also shown that the pretreatment of COPD patients with inhaled procaterol improves dynamic 
(A)

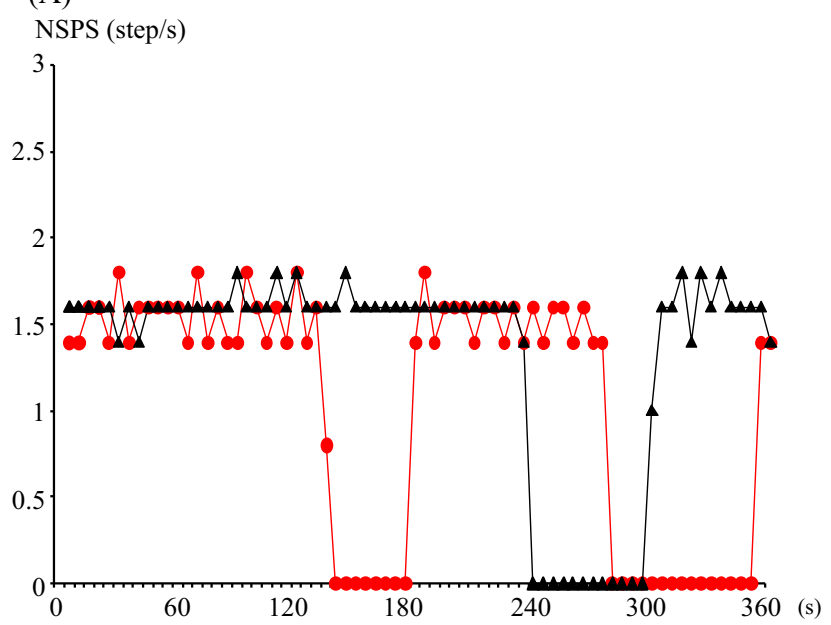

(B)

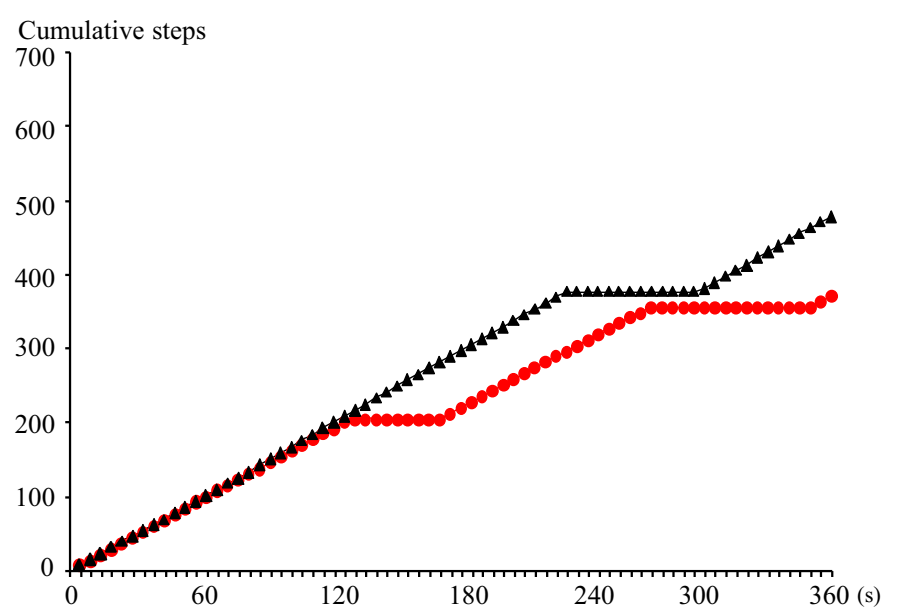

Figure 3. Determining the walking pattern and pace from the number of steps per second (NSPS) and the cumulative steps before and after the inhalation of procaterol. The patient was an 80-year-old man with COPD. Although the patient walked $177 \mathrm{~m}$ in the 6-min walk test (6MWT) with $114 \mathrm{~s}$ of rest before the inhalation of procaterol (closed circle), he could walk $249.5 \mathrm{~m}$ with $62 \mathrm{~s}$ of rest after the inhalation of procaterol (closed triangle) (A). The cumulative number of steps is shown as a function of time for each test (B). The slope indicates the walking pace. This patient walked at the same pace before and after the inhalation of procaterol, except for during rest periods.

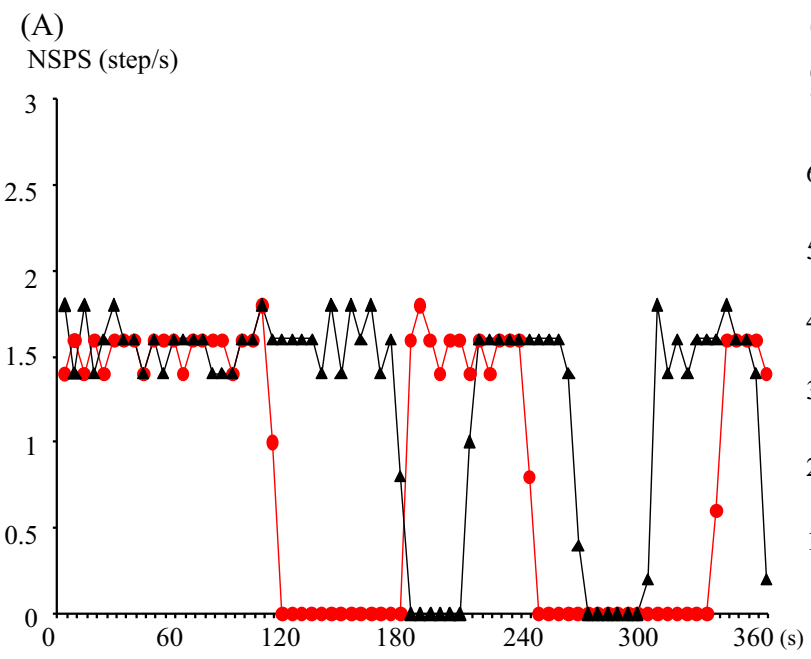

(B)

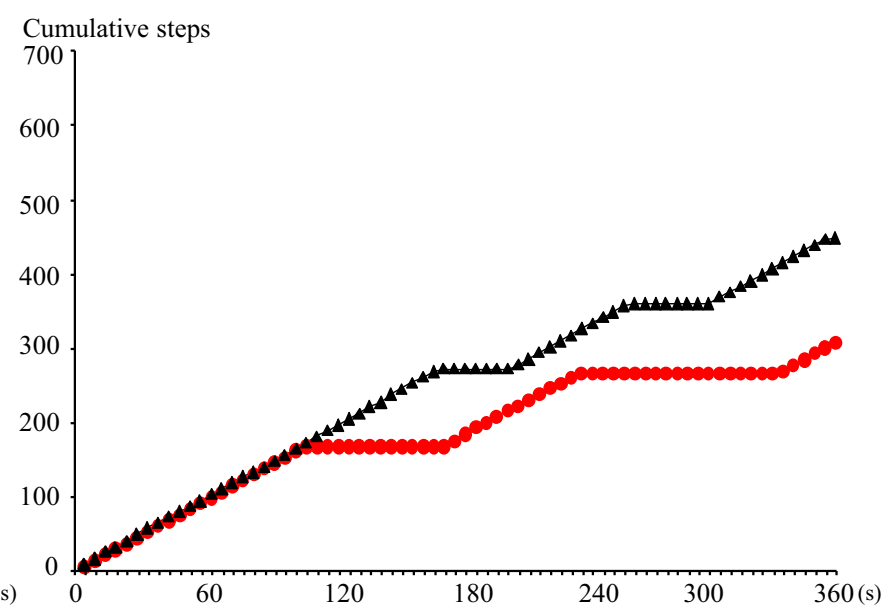

Figure 4. Determining the walking pattern and pace from the number of steps per second (NSPS) and the cumulative steps before and after the inhalation of procaterol. The patient was an 81-year-old man with COPD. Although the patient walked $125 \mathrm{~m}$ in the 6-min walk test (6MWT) with $158 \mathrm{~s}$ of rest before the inhalation of procaterol (closed circle), he could walk $188 \mathrm{~m}$ with $77 \mathrm{~s}$ of rest after the inhalation of procaterol (closed triangle) (A). The cumulative number of steps is shown as a function of time for each test (B). The slope indicates the walking pace. This patient walked at the same pace before and after the inhalation of procaterol, except for during rest periods.

lung hyperinflation and exercise tolerance (10, 11, 16-18). A previous report also indicated that the inhalation of formoterol increased the 6MWD (19). In addition, Fujimoto et al. reported that dynamic lung hyperinflation secondary to hyperventilation reduced the IC in COPD patients, while the IC was increased by the inhalation of procaterol (10). Moreover, the use of procaterol has been shown to improve both the exercise tolerance and health-related quality of life (16).
In the present study, the 6MWD was significantly increased after the inhalation of procaterol. It is reported that a $>30-\mathrm{m}$ increase in the 6MWD represents the minimal clinically important difference (MCID) for COPD patients $(1,2)$. The 6MWD changed by $>30 \mathrm{~m}$ following the inhalation of procaterol in 4 of the 9 COPD patients. In a graph of time versus cumulative steps, 3 of the 4 patients who showed an improvement of $>30 \mathrm{~m}$ after the inhalation of procaterol 
(A)

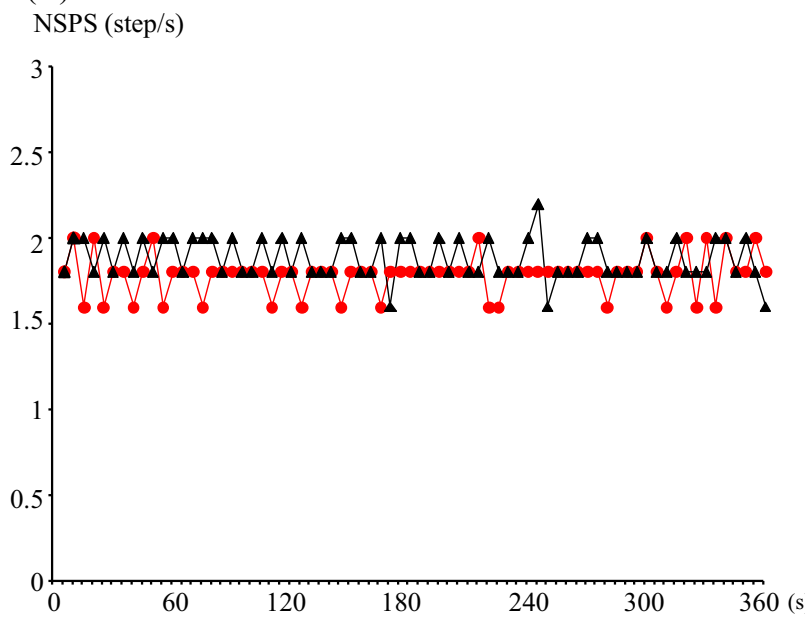

(B)

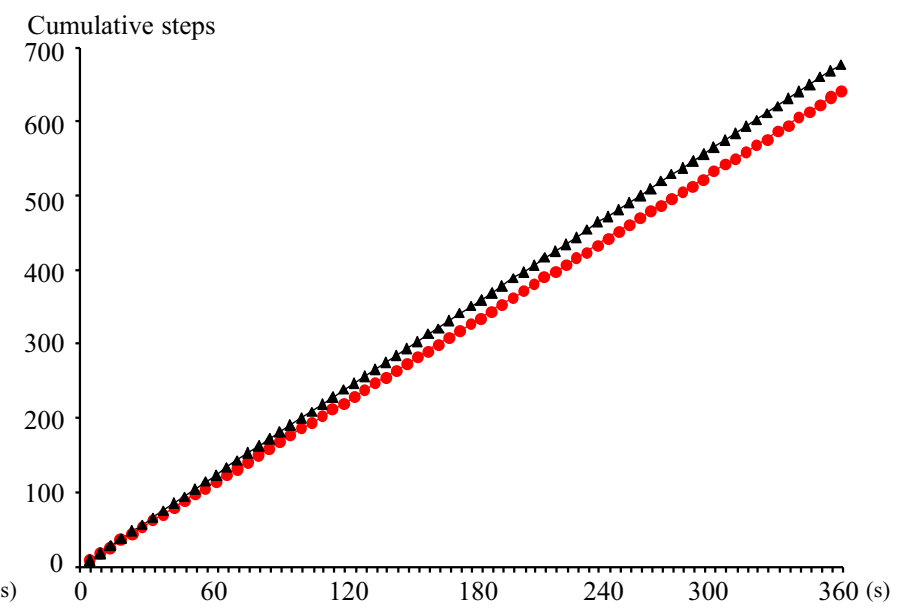

Figure 5. Determining the walking pattern and pace from the number of steps per second (NSPS) and the cumulative steps before and after the inhalation of procaterol. The patient was a 68-year-old man with COPD. Although the patient walked $356.5 \mathrm{~m}$ in a 6-min walk test (6MWT) without a rest before the inhalation of procaterol (closed circle), he could walk $422 \mathrm{~m}$ after the inhalation of procaterol (closed triangle) (A). The cumulative number of steps is shown as a function of time for each test $(B)$. The slope indicates that the walking pace was increased by the inhalation of procaterol.

showed a shorter total rest time, and the walking pace of one patient increased during the 6MWT. Thus, the increase in the 6MWD after the inhalation of procaterol might be a clinically relevant effect in 4 patients.

Although Golpe et al. found that exercise-related oxygen desaturation during the test was not an independent predictor in a multivariate analysis (20), the 6MWT has been reported to predict mortality in COPD patients (20-22). However, patients with moderate or severe COPD frequently rest during the 6MWT, as was noted in the present study, and exercise-related oxygen desaturation could improve after resting. In this study, the modified Borg scale after the 6MWT, the lowest $\mathrm{SpO}_{2}$, and the mean $\mathrm{SpO}_{2}$ were not significantly changed by the inhalation of procaterol. This suggests that our COPD patients walked as far as possible until they needed to rest during both tests, and recovered $\mathrm{SpO}_{2}$, resulting in no significant change in dyspnea or in the lowest or mean $\mathrm{SpO}_{2}$ values. Thus, it is important to take the rest time during the 6MWT into consideration when assessing the changes in $\mathrm{SpO}_{2}$ and desaturation during exercise. The previous reports did not clarify importance of a number of points, including the number of rests and the total resting time. The NSPS value, which decreases with rest during the 6MWT, can be used to evaluate the impact of resting in the $6 \mathrm{MWT}$. An analysis of the walking pattern and pace during the 6MWT would be useful in clinical studies on COPD medications.

The total steps walked during the $6 \mathrm{MWT}$ is reported to be useful for assessing the exercise capacity of patients with chronic heart disease $(23,24)$. We have developed a modified 6MWT, in which the walking pattern is examined by counting steps (12), allowing the walking pattern of COPD patients to be displayed on a graph of time versus NSPS. In addition, the walking pace could be displayed by a graph of time versus cumulative steps because $\operatorname{NSPS}_{k}(\mathrm{k}=1,2,3, \ldots, 72)$ shows a slope in 5-s (12). Moreover, it has been reported that a 3-dimensional accelerometer can accurately detect steps while a subject is walking $(23,24)$, and evaluate daily physical activity (25). If a pulse oximetry system that incorporates a 3-dimensional accelerometer that can accurately count steps is developed, the device could record $\mathrm{SpO}_{2}$ continuously, compute the NSPS, and plot a graph of time versus NSPS automatically to display the walking pattern during the 6MWT.

The present study is associated with several limitations, with the most obvious being its relatively small sample size. Furthermore, we did not investigate a random sample of COPD patients who showed a spectrum of disease severity or use a placebo. Further studies that include a placebo group will be needed to clarify the effects of pretreatment with inhaled procaterol on the exercise performance of COPD patients (26) and the usefulness of our new modified $6 \mathrm{MWT}$ with NSPS in assessing the response to drug treatment and the efficacy of pulmonary rehabilitation.

The authors state that they have no Conflict of Interest (COI).

\section{References}

1. Singh SJ, Puhan MA, Andrianopoulos V, et al. An official systematic review of the European Respiratory Society/American Thoracic Society: measurement properties of field walking tests in chronic respiratory disease. Eur Respir J 44: 1447-1478, 2014.

2. Holland AE, Spruit MA, Troosters T, et al. An official European Respiratory Society/American Thoracic Society technical standard: field walking tests in chronic respiratory disease. Eur Respir J 44: 1428-1446, 2014. 
3. ATS Committee on Proficiency Standards for Clinical Pulmonary Function Laboratories. ATS statement: guidelines for the sixminute walk test. Am J Respir Crit Care Med 166: 111-117, 2002.

4. Rasekaba T, Lee AL, Naughton MT, Williams TJ, Holland AE. The six-minute walk test: a useful metric for the cardiopulmonary patient. Intern Med J 39: 495-501, 2009.

5. Vestbo J, Hurd SS, Agustí AG, et al. Global strategy for the diagnosis, management, and prevention of chronic obstructive pulmonary disease: GOLD executive summary. Am J Respir Crit Care Med 187: 347-365, 2013.

6. Waschki B, Kirsten A, Holz O, et al. Physical activity is the strongest predictor of all-cause mortality in patients with COPD: a prospective cohort study. Chest 140: 331-342, 2011.

7. Waschki B, Spruit MA, Watz H, et al. Physical activity monitoring in COPD: compliance and associations with clinical characteristics in a multicenter study. Respir Med 106: 522-530, 2012.

8. O'Donnell DE, Revill SM, Webb KA. Dynamic hyperinflation and exercise intolerance in chronic obstructive pulmonary disease. Am J Respir Crit Care Med 164: 770-777, 2001.

9. Laveneziana P, Webb KA, Ora J, Wadell K, O’Donnell DE. Evolution of dyspnea during exercise in chronic obstructive pulmonary disease: impact of critical volume constraints. Am J Respir Crit Care Med 184: 1367-1373, 2011.

10. Fujimoto K, Yoshiike F, Yasuo M, et al. Effects of bronchodilators on dynamic hyperinflation following hyperventilation in patients with COPD. Respirology 12: 93-99, 2007.

11. Satake M, Takahashi H, Sugawara K, et al. Inhibitory effect of procaterol on exercise dynamic lung hyperinflation during the 6min walk test in stable patients with chronic obstructive pulmonary disease. Arzneimittelforschung 61: 8-13, 2011.

12. Burioka N, Imada A, Kiyohiro A, et al. Modified six-minute walk test: number of steps per second. Yonago Acta Medica 57: 61-63, 2014.

13. Borg G. Psychophysical scaling with applications in physical work and the perception of exertion. Scand J Work Environ Health 16 (Suppl 1): 55-58, 1990.

14. Solway S, Brooks D, Lacasse Y, Thomas S. A qualitative systematic overview of the measurement properties of functional walk tests used in the cardiorespiratory domain. Chest 119: 256-270, 2001.

15. Sciurba F, Criner GJ, Lee SM, et al. Six-minute walk distance in chronic obstructive pulmonary disease: reproducibility and effect of walking course layout and length. Am J Respir Crit Care Med
167: 1522-1527, 2003.

16. Hasegawa M, Dobashi K, Horie T, Wada N, Shirakura K. Influence of inhaled procaterol on pulmonary rehabilitation in chronic obstructive pulmonary disease. Respir Investig 50: 135-139, 2012.

17. Ohbayashi H, Adachi M. Pretreatment with inhaled procaterol improves symptoms of dyspnea and quality of life in patients with severe COPD. Int J Gen Med 5: 517-524, 2012.

18. Sukisaki T, Senjyu H, Oishi K, Rikitomi N, Ariyoshi K. Single dose of inhaled procaterol has a prolonged effect on exercise performance of patients with COPD. Physiother Theory Pract 24: 255-263, 2008.

19. Cazzola M, Biscione GL, Pasqua F, et al. Use of 6-min and 12min walking test for assessing the efficacy of formoterol in COPD. Respir Med 102: 1425-1430, 2008.

20. Golpe R, Pérez-de-Llano LA, Méndez-Marote L, Veres-Racamonde A. Prognostic value of walk distance, work, oxygen saturation, and dyspnea during 6-minute walk test in COPD patients. Respir Care 58: 1329-1334, 2013.

21. Pinto-Plata VM, Cote C, Cabral H, Taylor J, Celli BR. The 6-min walk distance: change over time and value as a predictor of survival in severe COPD. Eur Respir J 23: 28-33, 2004.

22. Casanova C, Cote C, Marin JM, et al. Distance and oxygen desaturation during the 6-min walk test as predictors of long-term mortality in patients with COPD. Chest 134: 746-752, 2008.

23. Jehn M, Schmidt-Trucksäess A, Schuster T, et al. Accelerometerbased quantification of 6-minute walk test performance in patients with chronic heart failure: applicability in telemedicine. J Card Fail 15: 334-340, 2009.

24. Jehn M, Prescher S, Koehler K, et al. Tele-accelerometry as a novel technique for assessing functional status in patients with heart failure: feasibility, reliability and patient safety. Int J Cardiol 168: 4723-4728, 2013.

25. Hataji O, Naito M, Ito K, Watanabe F, Gabazza EC, Taguchi O. Indacaterol improves daily physical activity in patients with chronic obstructive pulmonary disease. Int J Chron Obstruct Pulmon Dis 8: 1-5, 2013.

26. Aguilaniu B. Impact of bronchodilator therapy on exercise tolerance in COPD. Int J Chron Obstruct Pulmon Dis 5: 57-71, 2010.

The Internal Medicine is an Open Access article distributed under the Creative Commons Attribution-NonCommercial-NoDerivatives 4.0 International License. To view the details of this license, please visit (https://creativecommons.org/licenses/ by-nc-nd/4.0/).

(C) 2017 The Japanese Society of Internal Medicine http://www.naika.or.jp/imonline/index.html 